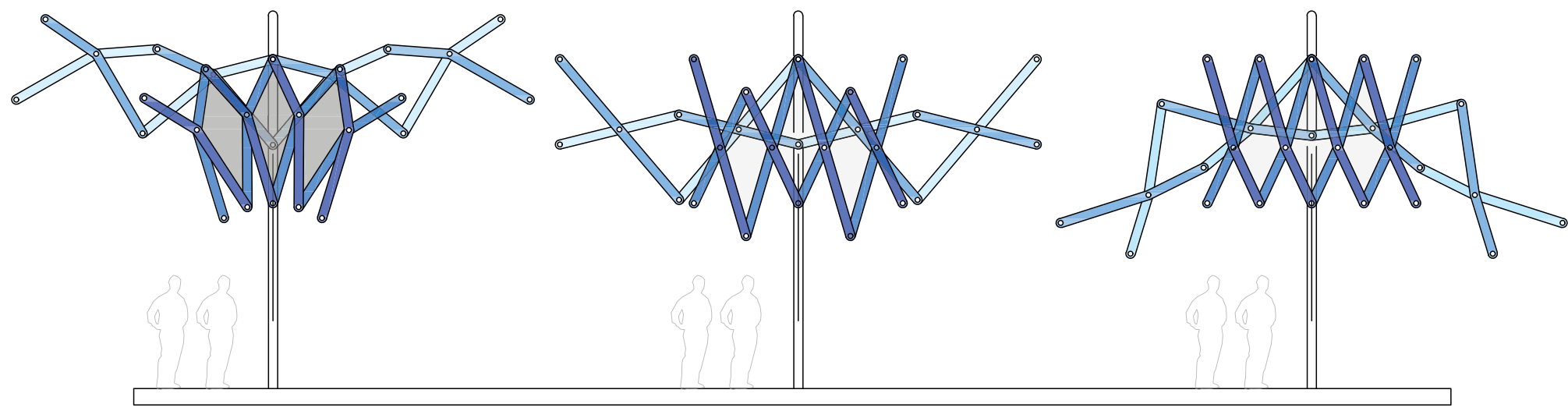

Scissor linkages of the same loop type (kite) resulting in different movements

\title{
- 3.3 Scissor linkages in the design of adaptive morphologies
}

Yenal Akgün, Feray Maden, Şebnem Gür, Gökhan Kiper, Koray Korkmaz, Engin Aktaş, Müjde Yar Uncu

In all periods of history, humans have tried to construct flexible buildings that are capable of adapting to ever-changing requirements and environmental conditions. In response to changing circumstances, they have proposed new solutions by incorporating movement into architecture. Thus, the concept of movement is not new to architecture. Indeed, the roots of the idea of capturing movement within a structure date back to ancient times. Simple nomadic tents built with flexible outer skins can be seen as the first example of adaptive structures used for protection against environmental extremes. ${ }^{1}$ The canvas sheets used for covering the roof of the Roman Coliseum, as another example, formed awnings to provide not only protection from the sun but also a breeze for the audience as it sloped down towards the centre to catch the wind. ${ }^{2}$

Adaptive roofs were used in demountable theatres as a protection against sun and rain in medieval times, even though the covering area was no larger than a few square metres. The concept of movement in architecture continued to play a significant role during the Renaissance. In the 18th century, awning constructions became widespread in Europe. In the 19th century, new design solutions and new technologies were introduced into architecture with the Industrial Revolution. New building materials such as cast iron, steel and glass offered the possibility and freedom to design new buildings and structures of a size, form and function unimaginable before. ${ }^{3}$ In the 20th century, technology became an increasingly important factor in enabling transformation and innovation in architecture and opened up new dimensions for adaptive forms of construction. The developments in architectural computing and material science have also facilitated the applications of adaptive/expandable structures. The growing relevance of these structures due to their advantages compared to conventional structures led to the development of different types of adaptive structures. Expandable structures comprise both a compact and a deployed form, usually comprising assemblies of rigid bodies connected by joints. They allow geometric transformations in order to satisfy practical requirements. As these structures are able to change their shapes from one configuration to another numerous times, they require dealing with kinematics (a sub-branch of mechanics) in addition to architectural and structural design. The complexity of design, construction and engineering processes for adaptive/ expandable structures necessitates an interdisciplinary design methodology with novel design approaches, theoretical principles and analytical methodologies.

\section{Scissor linkages: Applications in architecture} Scissor linkages are one of the most common types of adaptive/expandable structures. Since the Greek and Roman periods, these structures have been used in various applications in architecture and engineering as expandable roof structures, for shells and in furniture design. Scissor linkages are popular because they comprise very basic motion principles and can create both planar, beam-like expandable structures and expandable spatial shells with various geometries.
Compared to other types of adaptive structures (plate structures, strut-cable structures and membrane structures), scissor linkages are based on a simple system composed of scissor elements. To form a basic primary scissor unit, two rigid bars are connected to each other at an intermediate point through a pivotal connection that allows them to rotate freely about an axis perpendicular to their common plane. Planar and spatial expandable structures with different geometric shapes can be created by connecting multiple scissors to each other at their end nodes. A number of geometric principles and conditions of deployability must be provided to generate adaptive structures. A look at design methods described in existing literature provides an understanding of those principles and conditions, and forms the basis for two newly developed design methods with applications of scissor linkages.

\section{Design methods}

There are two basic methodologies for the design of expandable scissor linkages: the unit-based method and the loop assembly method. These methods utilise different approaches and have different advantages. Both are applicable for planar scissor beams and shells.

\section{Unit-based method}

The unit-based method allows the generation of an expandable scissor linkage by the serial multiplication of one of the scissor unit types. The scissor linkage is obtained by connecting the "primary 
a. Translational scissor unit

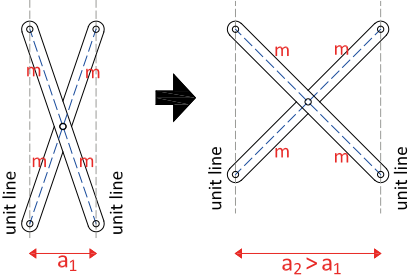

Translational planar scissor linkage

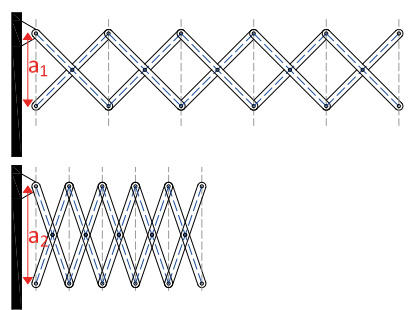

Common primary planar scissor units and their deployment

units" to each other through the terminal joints. The scissor linkages can be generated by multiplying one of the primary units or by a serial assembly of several sublinkages produced using a number of primary units. Various scissor linkages with different curvatures can be obtained in this way.

This method is based on the geometric characteristics of the primary scissor units. All types of primary scissor units use an imaginary line, the "unit line", between the corresponding upper and bottom ends of the bars. In the case where the unit lines remain parallel to each other during the deployment process, a "translational scissor unit" is obtained. A

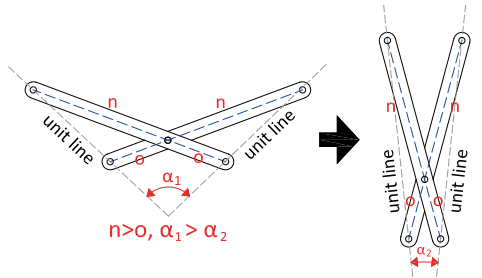

Polar planar scissor linkage

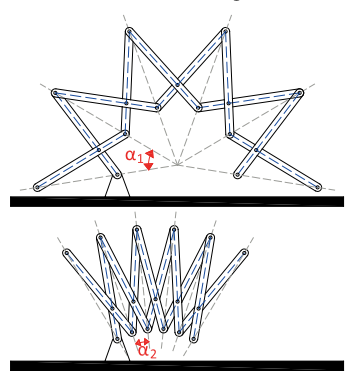

c. Angulated scissor unit

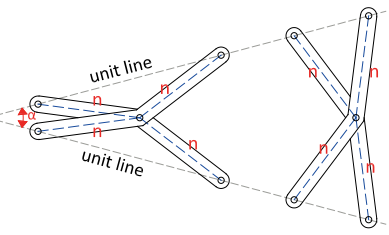

Planar scissor linkage with angulated scissor unit

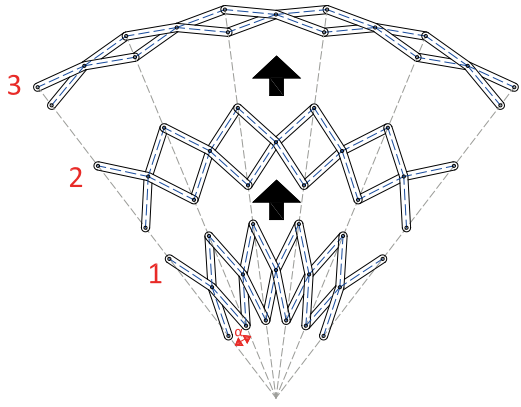

where the intention is to create primary planar or "polar scissor unit" is generated by connecting two straight bars with scissor hinges away from the midpoints of the bars. In this case, the unit lines intersect in one point and the segment angle $(\alpha)$ varies during the deployment. ${ }^{4}$ An "angulated scissor unit" is obtained by connecting two kinked bars instead of straight bars, with a kink angle between $90^{\circ}$ and $180^{\circ}$; the structure deploys radially and the segment angle $(\alpha)$ between two unit lines remains constant during the deployment.

The unit-based method is an inductive design process and very effective in cases where the target geometry of the linkage is a well-defined form, or spatial expandable geometries, like a planar arch, a linear beam or a dome-like shell. Spanish architect Emilio Pérez Piñero has pioneered the use of the unit-based method for expandable scissor linkages in architecture. He has developed many domes and spatial grids for functions like mobile theatres, pavilions and exhibition buildings. ${ }^{5}$ Following Piñero, Félix Escrig Pallares and his colleagues expanded the topic and presented the geometric conditions of deployability of scissor linkages composed of "translational and polar scissor units". They developed new spherical grid structures and several types a. Translational spatial scissor linkage

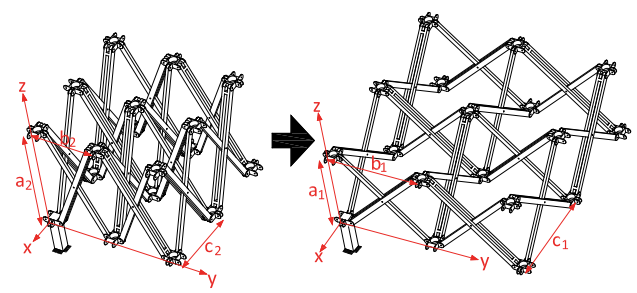

b. Polar spatial scissor linkage

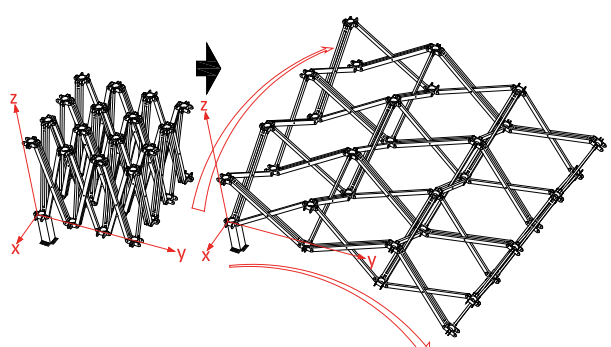

c. Planar linkage derived from angulated scissor unit

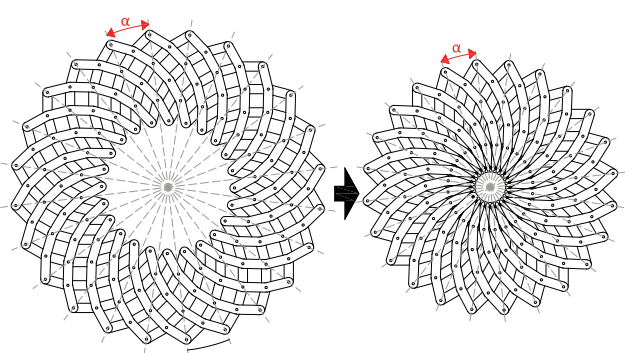

Spatial and planar scissor linkages and examples for their deployment

Brought to you by | provisional account Unauthenticated Download Date | 12/27/19 1:38 PM 


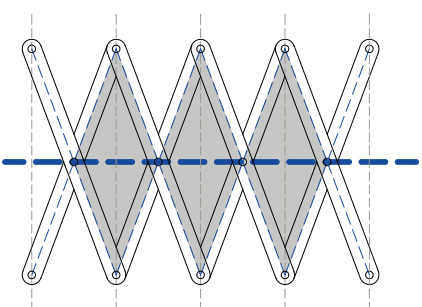

Rhombus loops

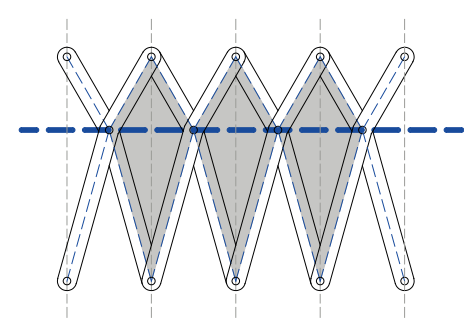

Kite loops

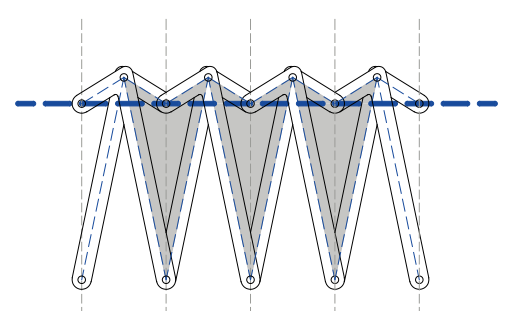

Dart loops

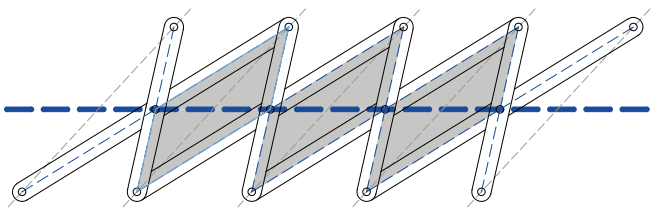

Parallelogram loops

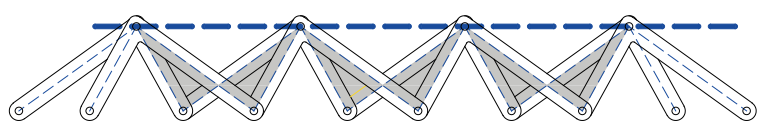

Anti-parallelogram loops

Loop types and the planar expanded scissor linkages that they produce

of expandable scissor structures, including quadrilateral expandable umbrella structures, expandable polyhedral structures and compactly folded cylindrical, spherical and geodesic structures. ${ }^{6,7}$

Chuck Hoberman made a remarkable invention on scissor linkages with the "angulated scissor unit". The discovery of this element extended the range of applications of scissor linkages by allowing the linkage to be deployed from its perimeter towards the centre, creating an opening at the centre. ${ }^{8,9} \mathrm{He}$ designed the Hoberman Arch, the Iris Dome and a number of other structures using the angulated element.

Many other researchers, among them Zhong You and Sergio Pellegrino ${ }^{10}$ as well as Charis Gantes, ${ }^{11}$ have explained the main principles, geometric properties and shape limitations of both planar and spatial expandable scissor linkages.

\section{Loop assembly method}

The other approach for defining expandable scissor linkages is the loop assembly method. This method allows several combinations between different loops and the desired geometry, thereby providing a practical way of designing scissor linkages with free-form curves. Rather than calculating individually each required link length and angle, a loop type is selected, and the desired number of loops are aligned on the curve. In the next step, the links and the primary scissor units are determined following the edges of the loops. In this way, the primary unit type/s is/are the result of this process, not its initial input.

This method was first used by Chuck Hoberman. He described the way in which identical angulated elements are paired to form angulated scissor pairs, with mechanisms that he termed "loop assemblies". At a later phase, in a lecture given at MIT, he described his construction of expanding polygons as an assembly of "hinged rhombuses".

The loop assemblies are formed by quadrilateral loops, which are the simplest movable loops. Quadrilaterals are called "rhombus", "kite", "dart", "parallelogram" and "anti-parallelogram", according to their geometry. If all four pairs of the quadrilateral loop are equal in length, it is defined as "rhombus". If the two adjacent pairs are equal, it is called "kite". A concave kite is called a "dart". A "parallelogram" is a convex quadrilateral with opposite sides parallel and equal in length; an "anti-parallelogram" is a quadrilateral in which each pair of opposite sides is equal but anti-parallel to the other two sides. By assembling such loops, expandable planar or spatial scissor linkages or shells can be obtained.

Different loop types can be applied to the same intended geometry. As described above, different types of loops can be located on a line, while the primary scissor unit and the deployment geometry differ according to the geometry of the loop type. Free-form planar geometries can be achieved by using different loop types. In this case, the deployment geometries are completely different. The loop assembly method is independent of the complexity of the intended form and can be adapted to any kind of planar expandable structure. The best way to proceed is to design the motion during expansion, or else decide intuitively which type of motion and loop is convenient for the intended design. Within the framework of the loop assembly method, even a specific single type of loop can provide different kinds of geometric behaviour. For example, kite loops of identical size can be applied to shade 


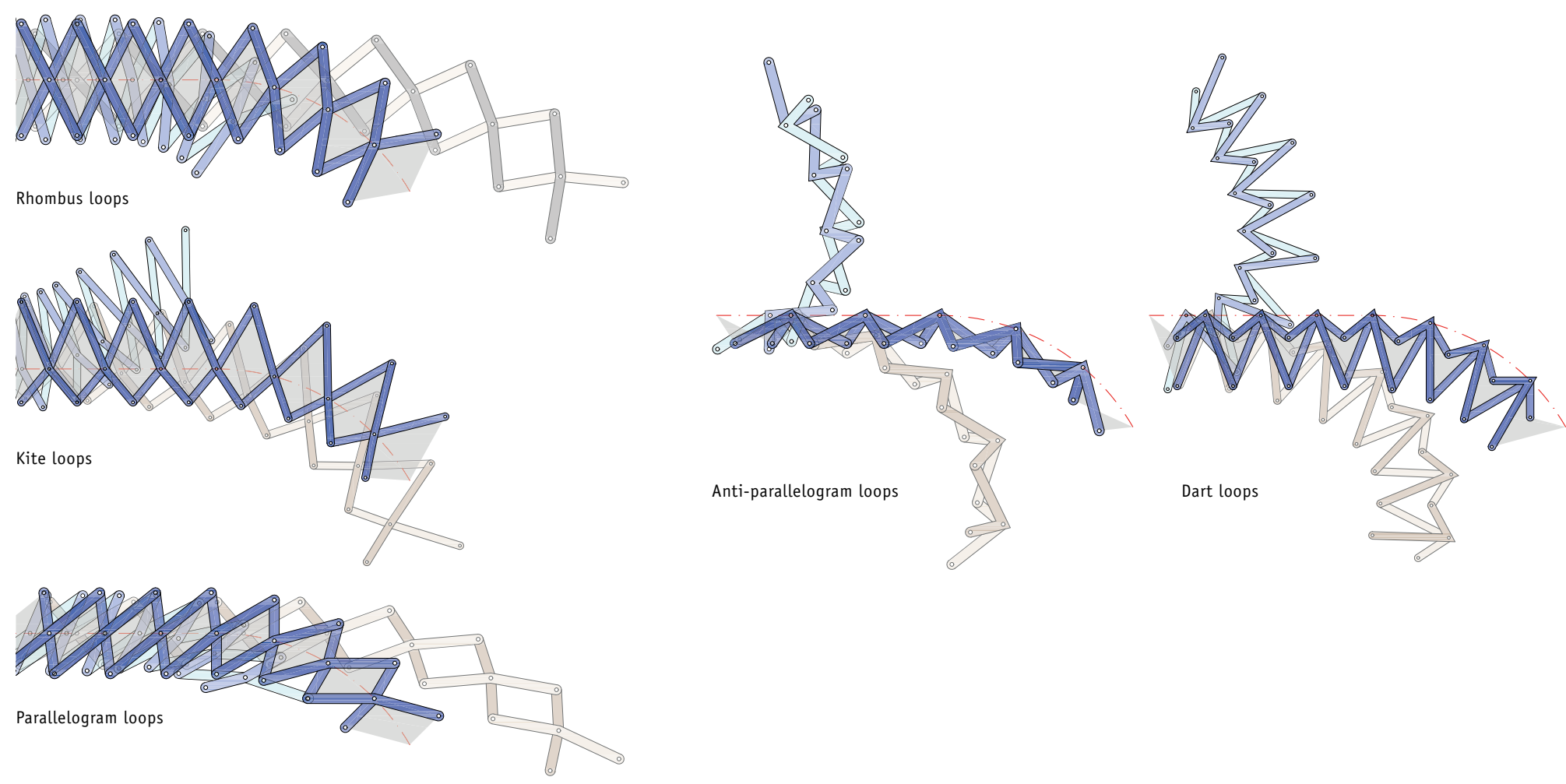

Expandable freeform scissor linkages with different loop types

structures with different assembly variations. Even though the loop geometry is identical, the ensuing primary scissor units as well as the deployment geometries differ significantly from each other.

\section{Summary}

Scissor linkages are capable of forming various expandable structures. Architects can benefit from this type of linkage especially for designing adaptive, movable, transformable shell structures and deployable beam-like structures. Product designers may benefit as well.

The two different methods described here convey the basic design approaches. The unit-based method is very effective for obtaining primary geometries like a dome, arch, circle or line, using serial multiplications and arrays of one of the scissor unit types presented here. The loop assembly method is more convenient when a final form, be it straight or free-form, is the main point of departure. In this case, unlike in the unit-based method, it is not necessary to opt for a specific scissor unit type and its dimensional constraints from the beginning. Designers can choose a type and number of loops and then define the scissor units following the loop sides. Since deployability is guaranteed by applying this method, the architect is free to choose the loop type most suitable for the functional needs and aesthetic concerns of the specific design. With the loop assembly method all loop alternatives can be assembled to scissor structures, their possible motions can be tested and evaluated in a short time, whereas the unit-based method is limited to a single type of motion that a specific unit can provide.
1 Tzonis, A.; Lefaivre, L.: Movement, Structure and the Work of Santiago Calatrava, Basel, 1995.

2 Zuk, W.; Clark, R. H.: Kinetic Architecture, New York, 1970.

3 Hitchcock, H. R.: Architecture: Nineteenth and Twentieth Centuries, London, 1987.

4 Maden, F.; Korkmaz, K.; Akgün, Y.: “Review of Planar Scissor Structural Mechanisms: Geometric Principles and Design Methods", Architectural Science Review, 54 (3), 2011, pp. 246-257.

5 Piñero, E.P.: “Project for a Mobile Theatre”, Architectural Design, 12, 1961, p. 570.

6 Escrig, F.: “Expandable Space Structures", Space Structures Journal, 2 (1), 1985, pp. 79-91.

7 Escrig, F.; Valcárcel, J.P.: “Geometry of Expandable Space Structures", International Journal of Space Structures, 8, 1993, pp. 71-84.

8 Hoberman, C.: Reversibly expandable doubly-curved truss structure, United States Patent No. US4942700A, 1990.

9 Hoberman, C.: “Mechanical Invention through Computation - Mechanism Basics", MIT Class 6.5080 Lecture notes, 2013, pp.37-47, http://courses.csail.mit. edu/6.S080/lectures/02_all.pdf.

10 You, Z.: Pellegrino, S.: “Foldable Bar Structures", International Journal of Solids and Structures, 15 (34), 1997, pp. 1825-1847.

11 Gantes, C.: Expandable Structures: Analysis and Design, Boston, 2001. 